\title{
Virasoro Action on Pseudo-Differential Symbols and (Noncommutative) Supersymmetric Peakon Type Integrable Systems
}

\author{
Partha Guha
}

Received: 6 June 2008 / Accepted: 26 August 2008 / Published online: 21 October 2008

(C) Springer Science+Business Media B.V. 2008

\begin{abstract}
Using Grozman's formalism of invariant differential operators we demonstrate the derivation of $N=2$ Camassa-Holm equation from the action of $\operatorname{Vect}\left(S^{1 / 2}\right)$ on the space of pseudo-differential symbols. We also use generalized logarithmic 2-cocycles to derive $N=2$ super $\mathrm{KdV}$ equations. We show this method is equally effective to derive CamassaHolm family of equations and these system of equations can also be interpreted as geodesic flows on the Bott-Virasoro group with respect to right invariant $H^{1}$-metric. In the second half of the paper we focus on the derivations of the fermionic extension of a new peakon type systems. This new one-parameter family of $N=1$ super peakon type equations, known as $N=1$ super $b$-field equations, are derived from the action of $\operatorname{Vect}\left(S^{1 \mid 1}\right)$ on tensor densities of arbitrary weights. Finally, using the formal Moyal deformed action of $\operatorname{Vect}\left(S^{1 \mid 1}\right)$ on the space of Pseudo-differential symbols to derive the noncommutative analogues of $N=1$ super $b$-field equations.
\end{abstract}

Keywords Pseudo-differential symbols $\cdot$ Super KdV · Camassa-Holm equation · Geodesic flow $\cdot$ Super $b$-field equations · Moyal deformation · Noncommutative integrable systems

Mathematics Subject Classification (2000) $17 \mathrm{~B} 68 \cdot 37 \mathrm{~K} 10 \cdot 58 \mathrm{~J} 40$

\section{Prelude to Noncommutative Integrable Systems}

Noncommutative geometry [5] extends the notions of classical differential geometry from differential manifold to discrete spaces, like finite sets and fractals, and noncommutative spaces which are given by noncommutative associative algebras. It was an idea of Descartes that we can study a space by means of functions on the space, in other words, the algebra

P. Guha $(\bowtie)$

Max Planck Institute for Mathematics in the Sciences, Inselstrasse 22, 04103 Leipzig, Germany

e-mail: partha.guha@mis.mpg.de

P. Guha

S.N. Bose National Centre for Basic Sciences, JD Block, Sector III, Salt Lake, Kolkata 700098, India 
of functions determines the space. Quantum physics suggests that some physical systems should be modeled by spaces on which functions are not commutative. In fact, $C^{*}$-algebras are natural models for the function algebras. In recent years it is appreciated that such noncommutative spaces retain a rich topology and geometry expressed in terms of $K$-theory and $K$-homology, as well as in finer aspects of the theory. The subject has also been approached from a more algebraic side with the advent of quantum groups and quantum homogeneous spaces [35].

Noncommutative geometry has recently been involved in a noncommutative gauge theory related to strings. Noncommutative spaces are characterized by the noncommutative coordinates

$$
\left[x_{i}, x_{j}\right]=i \theta^{i j},
$$

where $\theta^{i j}$ are real constants. During the last few years there has been a steady growth in the interest in noncommutative geometry, which appears in string theory in several ways. Much attention has been paid also to field theories on noncommutative spaces and especially Moyal deformed [38] space-time. This theory appears as certain limits of strings, $D$-branes and $M$-theory.

A remarkable feature of many $(1+1)$-dimensional PDEs that arise in geometry and mechanics is their explicit solvability based on some symmetries or a nonlinear transformation of variables. In the Hamiltonian settings, we use the notion of Liouville integrability to describe integrable systems. Liouville integrability means that there exists a regular foliation of the phase space by invariant manifolds such that the Hamiltonian vectors fields associated to the invariants of the foliation span the tangent distribution. A resurgence of interest in integrable systems came with the discovery of the Korteweg-de Vries (KdV) equation in late sixties. The KdV equation falls into the category of infinite dimensional integrable Hamiltonian systems. It is known that both the KdV [41] and the Camassa-Holm (CH) [37] equations are geodesic flows on the Bott-Viarsoro group [27]. In this paper we consider the noncommutative version of several infinite dimensional systems. There are several beautiful mathematical methods [11] exit to construct classical integrable systems, unfortunately no such generalized method exist for noncommutative integrable systems.

\subsection{NC Integrable Systems and Its Connection to Neighbouring Fields}

In most recent development of gauge theory, a central role is played by nonperturbative regimes, such as instantons, vortices and monopoles. These solitonic classical field configuration generically arise in a BPS sector of the theory. Noncommutative gauge theories are naively realized from ordinary commutative theories just by replacing all products of the fields with $\star$ product. String theory proposed a new non-commutative gauge theories that describe the dynamics of branes. Studying classical solutions of noncommutative field theories also establish the solitonic nature of $D$-branes in string theory [23, 31, 45]. It turns out that the Moyal deformed field theories have a much richer spectrum of soliton solution than their commutative parts. For example [31, 32], the Ward sigma model, which is integrable in $2+1$ dimensions and features exact multi-soliton configurations, can be Moyal deformed without loosing its integrability and it yields a novel class of abelian solitons, which exist even in the $U$ (1) case. The deformed supersymmetric Ward solitons (cf. [32]) are superextensions of the bosonic noncommutative Ward solitons as well as deformation of commutative Ward solitons. The case of a general non-Abelian gauge group is dealt with a construction of a Seiberg-Witten map [44], i.e. a map which connects the gauge theory on the noncommutative space with gauge theory on a commutative space and for this purpose the $\star$-product formalism is used. 
Integrable systems usually display localized classical solutions known as solitons. Once again, noncommutative deformation enormously enhances the spectrum of solitons. Several classical integrable models have been generalized to noncommutative spaces [10, 21]. Also, under the Moyal deformation, the self-dual Yang-Mills equation is considered to preserve the integrability in the same sense as in commutative cases and contain new solutions special to noncommutative spaces [39]. Noncommutative KdV and nonlinear Schrödinger equations are derived from the reduction of self dual Yang-Mills equation [22, 33] and other methods [13-16]. There exist a method, namely the bicomplex method [10], to yield noncommutative integrable equations which have many conserved quantities. Certainly all these equations are derived formally from the Lax representation by replacing ordinary product by $\star$ product.

Noncommutative extension of integrable systems such as the KdV equation, the super $\mathrm{KdV}$ equation are also one of the hot topics in noncommutative geometry and physics. In fact, some time ago Kupershmidt [29] considered a generalization of the Moyal approach to the problem of quantization of classical integrable dynamical systems. The nature of these noncommutative integrable equations are strange. They do not have good integrable property and on top of that Noncommutative extension of $(1+1)$-dimensional equations introduces infinite number of time derivatives. But they do possess the existence of infinite number of conserved quantities which are widely accepted as definition of complete integrability of underlying equations.

\subsection{Motivation, Result and Plan}

The noncommutative generalization of a given integrable systems is not unique. The symmetric structure plays an important characteristic feature. Therefore, preserving the symmetry structure of a theory should be the first criterion to consider when implementing its deformation. There are several methods to construct such noncommutative extension of integrable systems. By deforming the Lax equation (for example, [21]) one can derive such equations. This is a bit ad hoc formalism and does not incorporate geometry. Furthermore, many noncommutative integrable systems are obtained from the reductions of the NC SDYM equation. Again, none of them are related to Lie theoretic method. Indeed, in this paper we travel in quest for Lie theoretic formulation of noncommutative integrable systems through a true wilderness.

Recently a deformation of the algebra of diffeomorphism is constructed by Wess and coworkers [2] for canonically deformed spaces with constant deformation parameter $\theta$. Using this method we constructed the noncommutative version of periodic $\mathrm{KdV}$ and the Burgers equation [18], and this gives us the second method for the construction of Moyal deformed integrable systems. Although this method is far more geometrical than Lax equation method but certainly we faced problems for not having a proper (noncommutative) Hamiltonian formalism, since infinite time derivatives hidden inside the $\star$-product. One must remember that the deformation theory is a homotopy theory and does not incorporate dynamics easily.

The motivation of this present article to offer another interesting method of construction of noncommutative integrable systems. This method has been sketched in our earlier papers $[16,17,20]$. We embed the vector field and its dual to pseudodifferential symbols on $S^{1}$ $[42,43]$. These are functions on cotangent bundle. We use logarithmic 2-cocycle [26, 28] to derive the dispersion term. Therefore we lift the systems on the space of pseudodifferential symbols where the natural action of vector field on its dual is given by Poisson action. Thus coadjoint action [16] of Virasoro algebra on its dual can be manifested in a simple manner. 
In this paper we apply Grozman [15, 34] programme of invariant bilinear differential operators on tensor fields to compute this action. It is shown that this scheme can be applied to the $N=2$ supersymmetry theory [47] provided one must take care of anticommutative properties of fermions. In this work we reexamine the proof of $N=2$ supersymmetric KdV equation using Grozman prescription. We also derive the $N=2$ supersymmetric Camassa-Holm equation from our method. In both cases we derive explicit representation of the second Hamiltonian structures.

By deforming the Poisson action to Moyal action we obtain the quantum integrable systems. In fact Kupershmidt [29] also proposed such method to quantize integrable systems. We argue that this method is deeply rooted inside the Moyal-Weyl-Wigner formalism. The Moyal algebra $\left(C^{\infty}(M),\{., .\}_{\text {Moyal }}\right)$ is an algebra of quantum observables and it can be continuously reduced the Poisson algebra $\left(C^{\infty}(M),\{.,\}_{P B}\right)$ of classical observables. Using this new approach we derive various integrable and superintegrable systems. Using this approach we derive various integrable and superintegrable systems. In this method we are able to quantize a completely new and exotic super $b$-field equation. This new class of partial differential equations recently obtained by Degasperis Holm and Hone [7, 8] using the asymptotic integrability method. The second member of this one-parameter family of PDEs is called Degasperis-Procesi equation [6]. Degasperis et al. proved the exact integrability of the new equation by constructing its Lax pair and explain its relation to a negative flow in the Kaup-Kupershmidt hierarchy via a reciprocal transformation.

The goal of this paper is to add supersymmetry to the noncommutative systems. Devchand and Schiff [9] showed that the fermionic extension of the Camassa-Holm equation arises as a geodesic flows of an invariant $H^{1}$-metric on the group of superconformal transformations. In this paper we focus on to fermionic extension of the $b$-field equations. We derive this equation from the action of $\operatorname{Vect}\left(S^{1 \mid 1}\right)$ on tensor densities of weight $b$ form embedded in $\psi D\left(S^{1}\right)$. We also derive the noncommutative version of these system of equations. Therefore we also able to quantize a completely new and exotic super $b$-field equation. The advantage of this method is that we can avoid the quantization of coadjoint orbit to derive quantum (or noncommutative ) integrable systems.

This paper is organized as follows: In Sect. 2 we give a brief description of pseudodifferential symbols on $S^{1}$ and the construction of $\mathrm{KdV}$ equations. We introduce generalized Souriau cocycle in Sect. 3. In this section we demonstrate the construction of the $N=2$ super $\mathrm{KdV}$ equation. Section 4 is devoted to the construction of the $N=2$ supersymmetric Camassa-Holm equations. We also show its geodesic connection to superconformal group. Section 5 is devoted to the construction of the $b$-field and super $b$-field equations. Finally, the noncommutative (or Moyal deformed) analogue of the super peakon type equations are given in Sect. 6.

\section{Background: Pseudodifferential Symbols on $S^{\mathbf{1}}$ and KdV Equation}

The ring of pseudodifferential symbols on $S^{1}, \Psi D\left(S^{1}\right)$, is defined to be the ring of formal Laurent series $\sum_{k \geq k_{0}} f_{k}(x) \xi^{k}$ over $C^{\infty}\left(S^{1}\right)$ with finite number of positive powers. There are two differentiations defined in this ring $\Psi D\left(S^{1}\right)$ :

$$
\partial_{\xi}: \sum_{k} f_{k} \xi^{k} \longmapsto \sum_{k} k f_{k} \xi^{k-1}, \quad \partial: \sum_{k} f_{k} \xi^{k} \longmapsto \sum_{k} f_{k}^{\prime} \xi^{k}
$$


These differentiations may be used to define the symbolic multiplication on the ring by setting

$$
(F \circ G)=\sum_{k=0}^{\infty} \frac{1}{k !}: \frac{\partial^{k} F}{\partial \xi^{k}}(x, \xi) \frac{\partial^{k} G}{\partial x^{k}}(x, \xi): .
$$

Here : : is called the Normal Ordering; it is defined by

$$
: f(x) \xi^{k} g(x) \xi^{l}:=f(x) g(x) \xi^{k+l} .
$$

This multiplication rules yields an associative and a Lie algebra operation on the ring. The commutator and the residue map are defined, respectively, by setting $[F, G]=F \circ G-G \circ F$ and res $: \psi D\left(S^{1}\right) \rightarrow C^{\infty}\left(S^{1}\right)$. The "trace operation" is defined by

$$
\operatorname{Tr}(F)=\int_{S^{1}} \operatorname{res} F d x=\int_{S^{1}} f_{-1} d x .
$$

The main property of the residue (or trace) is for $F, G \in \psi D\left(S^{1}\right)$,

$$
\operatorname{Tr}([F, G])=\int \operatorname{res}[F, G] d x=0 .
$$

The embedding of the vector field $\pi(f(x) \partial)=f(x) \xi$ enables one to pass from the Virasoro algebra to the algebra of groups of area preserving diffeomorphisms. The dual of the vector field is identified with $u(x) \xi^{-2} \in \mathcal{F}_{2}$.

This algebra of embedded vector fields can be extended via logarithmic cocycle.

Theorem 1 (Kravchenko and Khesin) Let $F$ and $G$ be pseudo differential symbols on the circle. The nontrivial central extension $\psi D\left(S^{1}\right)$ is given by the cocycle

$$
c(F, G)=\int \operatorname{res}([\ln \xi, F], G) .
$$

The restriction of Kravchenko-Khesin cocycle [28] to the subalgebra of vector fields is the Gelfand-Fuchs cocycle of the Virasoro algebra. This follows from a simple calculation

$$
\begin{aligned}
c(f(x) \xi, g(x) \xi) & =\int \operatorname{res}([\ln \xi, f(x) \xi] \circ g(x) \xi) \\
& =\int \operatorname{res}\left(f^{\prime}(x)-\frac{f^{\prime \prime}(x)}{2} \xi^{-1}+\frac{f^{\prime \prime \prime}(x)}{3} \xi^{-2}+\cdots\right) g(x) \xi \\
& =\frac{1}{6} \int f^{\prime \prime \prime}(x) g(x) d x .
\end{aligned}
$$

The term containing the $\xi^{-2} \in \mathcal{F}_{2}$ in the expansion

$$
\mathbb{S}\left(f(x) \frac{d}{d x}\right)=f^{\prime \prime \prime}(x) \xi^{-2}
$$

is called the Souriau cocycle of the Virasoro algebra. One should note that the space of tensor density of degree 2 is the regular dual of $\operatorname{Vect}\left(S^{1}\right)$. 


\subsection{Construction of Full KdV Equation}

Our goal is to give a geometric formulation of noncommutative integrable systems. Let us reformulate the Euler-Poincaré flow [25] in a following form:

Definition 1 Let $d H$ be the gradient of the Hamiltonian function $H(u)$. The Euler-Poincaré equation induced by the action of $\operatorname{Vect}\left(S^{1}\right)$ on its dual is:

$$
u_{t}=a d_{(d H) \xi}^{*} u \xi^{-2} \equiv\left\{(d H) \xi, u \xi^{-2}\right\} .
$$

Theorem 2 The Euler-Poincaré flow which is induced by the action of $\operatorname{Vect}\left(S^{1}\right)$ on the extended space of $\psi D\left(S^{1}\right)$ is defined as

$$
u_{t}=\left\{\frac{\delta H}{\delta u} \xi, u \xi^{-2}\right\}+\left(\frac{\delta H}{\delta u}\right)^{\prime \prime \prime} \xi^{-2} .
$$

Let $H=\frac{1}{2} \int_{S^{1}} u^{2} d x$. This yields the KdV equation

$$
u_{t}+u_{x x x}+3 u u_{x}=0 .
$$

Proof The action of $\operatorname{Vect}\left(S^{1}\right)$ on the extended $\Psi D\left(S^{1}\right)$ comes from two sources; (a) the original action of $\operatorname{Vect}\left(S^{1}\right)$ on $\Psi D\left(S^{1}\right)$ and (b) Souraiu term. The term $f^{\prime \prime \prime}(x) \xi^{-2}$ in the following equation is the Souraiu term.

The coadjoint action of $\operatorname{Vect}\left(S^{1}\right)$ on its dual $u(x) \xi^{-2}$ is given by

$$
\begin{aligned}
a d_{f(x) \xi}^{*} u(x) \xi^{-2} & =\left\{f(x) \xi, u(x) \xi^{-2}\right\} \\
& =-\left(\frac{\partial}{\partial \xi}(f(x) \xi)\right) \frac{\partial}{\partial x}\left(u \xi^{-2}-\frac{\partial}{\partial x}(f(x) \xi)\right) \frac{\partial}{\partial \xi}\left(u \xi^{-2}\right) \\
& =-\left(f u^{\prime}+2 f^{\prime} u\right) \xi^{-2}
\end{aligned}
$$

Therefore the action of $\operatorname{Vect}\left(S^{1}\right)$ on the extended $\Psi D\left(S^{1}\right)$ is given by

$$
\begin{aligned}
\widetilde{a} d_{f(x) \xi}^{*}\left(u(x) \xi^{-2}, f^{\prime \prime \prime}(x) \xi^{-2}\right) & =\left\{f(x) \xi, u(x) \xi^{-2}\right\}+f^{\prime \prime \prime} \xi^{-2} \\
& =\left(f u^{\prime}+2 f^{\prime} u+f^{\prime \prime \prime}\right) \xi^{-2}
\end{aligned}
$$

One can easily use (8) to compute the second Hamiltonian operator $\mathcal{O}_{K d V}$ of the KdV equation which corresponds to the action of $\operatorname{Vect}\left(S^{1}\right)$ on the extended $\psi D\left(S^{1}\right)$ given by:

$$
\mathcal{O}_{K d V}=\partial u+u \partial+\partial^{3} .
$$

\section{The $N=2$ Neveu-Schwarz Super Algebra and Construction of the $N=2$ Super KdV Equation}

Let us introduce the Neveu-Schwarz superalgebra. Consider the space of $-1 / 2$-tensor densities $\mathcal{F}_{1 / 2}$ on $S^{1}$. There exists a natural Lie superalgebra structure on the space of $\operatorname{Vect}\left(S^{1}\right) \oplus \mathcal{F}_{1 / 2}$. The anticommutator

$$
[,]_{+}: \mathcal{F}_{1 / 2} \otimes \mathcal{F}_{1 / 2} \longrightarrow \operatorname{Vect}\left(S^{1}\right) \in \mathcal{F}_{1}
$$


is just the product of tensor densities:

$$
\left[\psi(x)(d x)^{-1 / 2}, \phi(x)(d x)^{-1 / 2}\right]_{+}:=\psi(x) \phi(x) \frac{d}{d x} .
$$

The space of $-1 / 2$ densities on $S^{1}$ can be periodic or anti-periodic

$$
\phi(x)(d x)^{-1 / 2}, \quad \phi(x+2 \pi)= \pm \phi(x),
$$

known as Ramond space and Neveu-Schwarz space respectively and these spaces of $-1 / 2$ tensor densities are $\operatorname{Vect}\left(S^{1}\right)$-module. The Neveu-Schwarz space is customarily assigned by $\mathcal{F}_{1 / 2}^{-}$.

Definition 2 Let $\mathcal{F}_{-3 / 2}^{-}$be the space of antiperiodic $3 / 2$ densities. The space

$$
\mathfrak{g}=\operatorname{Vect}\left(S^{1}\right) \oplus \mathcal{F}_{1 / 2}^{-} \oplus \mathcal{F}_{1 / 2}^{-} \oplus C^{\infty}\left(S^{1}\right)
$$

defines a $N=2$ Lie superalgebra structure, it is known as $N=2$ Neveu-Schwarz algebra $\mathfrak{g}$. The (regular) dual space of the Neveu-Schwarz algebra is given as

$$
\mathfrak{g}^{*}=\mathcal{F}_{-2} \oplus \mathcal{F}_{-3 / 2}^{-} \oplus \mathcal{F}_{-3 / 2}^{-} \oplus \mathcal{F}_{-1}^{-}
$$

Since we consider only the Neveu-Schwarz algebra so we drop the '-' sign from $\mathcal{F}$.

Let us now embed the Neveu-Schwarz algebra into space of pseudodifferential symbols. We obtain following mappings for algebra

$$
\left(f(x) \frac{d}{d x}+\phi_{i}(x)(d x)^{-1 / 2}+g(x)\right) \longmapsto\left(f(x) \xi+\phi_{i}(x) \xi^{1 / 2}+g(x)\right),
$$

and the corresponding dual is given by

$$
\left(u(x) d x^{2}+\eta_{i}(x)(d x)^{3 / 2}\right)+w(x)(d x) \longmapsto\left(u(x) \xi^{-2}+\eta_{i}(x) \xi^{-3 / 2}+w(x) \xi^{-1}\right),
$$

where sum over repeated index is implied. One must note that $N=2$ superconformal algebra has two fermionic fields, denoted by $\phi_{1}$ and $\phi_{2}$, and two bosonic fields $u(x)$ and $w(x)$.

Proposition 1 (Grozman) The classification of invariant bilinear differential operators on tensor fields is due to P. Grozman. Let us recall the zeroth-order and the first order cases:

1. There exists a suitable constant so that a zeroth-order operator $\mathcal{F}_{\nu} \otimes \mathcal{F}_{\mu} \longrightarrow \mathcal{F}_{v+\mu}$ has the form

$$
\phi(x)(d x)^{v} \otimes u(x)(d x)^{\mu} \longmapsto k \phi(x) u(x)(d x)^{v+\mu} .
$$

2. Every first order operator $\mathcal{F}_{\nu} \otimes \mathcal{F}_{\mu} \longrightarrow \mathcal{F}_{v+\mu+1}$ is given by

$$
\left\{\phi(x)(d x)^{v}, u(x)(d x)^{\mu}\right\}=\left(v \phi(x) u^{\prime}(x)-\lambda \phi^{\prime}(x) u(x)\right)(d x)^{\nu+\mu+1} .
$$

For every $(\nu, \mu) \neq(0,0)$, the operator (11) is the only Diff $\left(S^{1}\right)$ operator, otherwise there are two linearly independent operators $\phi d(u)$ and $d(\phi) u$, where $d$ is the de Rham differential operator. 
Let us study the action of the Neveu-Schwarz algebra on $u(x) \xi^{-2}+\eta(x) \xi^{-3 / 2}$. The computation of this action is based on the Grozman formalism [15] of bilinear invariant differential operators on tensor densities.

Lemma 1 The Hamiltonian operator $\mathcal{O}$ corresponding to the action of the $N=2$ NeveuSchwarz algebra on $u(x) \xi^{-2}+\eta_{i}(x) \xi^{-3 / 2}+w(x) \xi^{-1} \in \Psi D\left(S^{1}\right)$ yields

$$
\mathcal{O}=\left(\begin{array}{cccc}
u \partial+\partial u & \frac{1}{2} \partial \eta_{1}+\eta_{1} \partial & \frac{1}{2} \partial \eta_{2}+\eta_{2} \partial & w \partial_{x} \\
\partial \eta_{1}+\frac{1}{2} \eta_{1} \partial & -\frac{1}{2} u & \frac{1}{2} \partial w+\frac{1}{2} w \partial & \frac{k_{4}}{2} \eta_{2} \\
\partial \eta_{2}+\frac{1}{2} \eta_{2} \partial & -\frac{1}{2} w \partial-\frac{1}{2} \partial w & -\frac{1}{2} u & \frac{k_{3}}{2} \eta_{1} \\
\partial w & \frac{k_{1}}{2} \eta_{2} & \frac{k_{2}}{2} \eta_{1} & 0
\end{array}\right) .
$$

Proof Let $\mathcal{F}_{\lambda}$ be the space of $-\lambda$-densities on $S^{1}$. Any zeroth-order differential operator is the operator of multiplication by a $(\mu-\lambda)$ density:

$$
\phi(x)(d x)^{\mu-\lambda}: u(x)(d x)^{\lambda} \longmapsto \phi(x) u(x)(d x)^{\mu} .
$$

In standard Darboux coordinates this can be written as:

$$
\phi(x) \xi^{-\mu+\lambda}: u(x) \xi^{-\lambda} \longmapsto \phi(x) u(x) \xi^{-\mu} .
$$

Moreover, there exists a pair of duals $\left(\eta_{1}(d x)^{3 / 2}, \eta_{2}(d x)^{3 / 2}\right)$ corresponding to a pair $-1 / 2$ densities functions $\left(\phi_{1}(x)(d x)^{-1 / 2}, \phi_{2}(x)(d x)^{-1 / 2}\right)$. It is clear that $\phi_{1}(x)$ acts on its dual $\eta_{1}$ in an obvious way and $\eta_{2}$ by the principle of zeroth-order differential operator.

Hence, the action of $f(x) \xi+\phi_{i}(x) \xi^{1 / 2}+g(x) \in \operatorname{sect}\left(S^{1}\right)$ on its dual is:

$$
\begin{aligned}
& a d_{f(x) \xi+\phi_{i}(x) \xi^{1 / 2}+g(x)}^{*}\left(u(x) \xi^{-2}+\eta_{i}(x) \xi^{-3 / 2}+w(x) \xi^{-1}\right) \\
& =\left\{f(x) \xi, u(x) \xi^{-2}+\eta_{i}(x) \xi^{-3 / 2}+w(x) \xi^{-1}\right\}+\left\{\phi_{1}(x) \xi^{1 / 2}, \eta_{1}(x) \xi^{-3 / 2}+w(x) \xi^{-1}\right\} \\
& \quad+\left\{\phi_{2}(x) \xi^{1 / 2}, \eta_{2}(x) \xi^{-3 / 2}\right\}-\left\{\phi_{2}(x) \xi^{1 / 2}, w(x) \xi^{-1}\right\}+\left\{g(x), w(x) \xi^{-1}\right\} \\
& \quad+\frac{k}{2} \phi_{i}(x) u(x) \xi^{-3 / 2} \\
& \quad+\frac{k_{1}}{2} \phi_{1}(x) \eta_{2} \xi^{-1}+\frac{k_{2}}{2} \phi_{2}(x) \eta_{1}(x) \xi^{-1}+\frac{k_{3}}{2} g(x) \eta_{1} \xi^{-3 / 2}+\frac{k_{4}}{2} g(x) \eta_{2} \xi^{-3 / 2}
\end{aligned}
$$

where the last expression follows from the definition of zeroth operator and we have chosen $k=-\frac{1}{2}$. Therefore, we obtain the Hamiltonian operator from this expression, thereby completing the proof:

$$
\begin{aligned}
= & \left(f u^{\prime}+2 f^{\prime} u+\frac{1}{2} \phi_{i} \eta_{i}^{\prime}+\frac{3}{2} \phi_{i}^{\prime} \eta_{i}+f w^{\prime}+f^{\prime} w\right) \xi^{-2} \\
& +\left(f(x) \eta^{\prime}+\frac{3}{2} f^{\prime} \eta_{i}(x)-\frac{1}{2} \phi_{i}(x) u(x)+(-1)^{i-1} \frac{1}{2}\left(\phi_{i} w^{\prime}+\phi_{i} w^{\prime}\right)\right. \\
& \left.+\frac{k_{3}}{2} g(x) \eta_{1}+\frac{k_{4}}{2} g(x) \eta_{2}\right) \xi^{-3 / 2} \\
& +\left(g(x) w^{\prime}(x)+\frac{k_{1}}{2} \phi_{1} \eta_{2}+\frac{k_{2}}{2} \phi_{2}(x) \eta_{1}(x)\right) \xi^{-1} .
\end{aligned}
$$


Comment One must careful to apply Grozman prescription for extended supersymmetry case. In this situation we encounter multiple fermions. Thus the action should respect the ordering of fermions. This does not appear in $N=1$ case, since we just play with a single fermion.

Remark All these constants $k$ and $k_{i} \mathrm{~s}$ are free parameters. These play an important role in integrability. For $N=1$ case we have just one parameter $k$ and its value directly connected to nature of the system. It can be shown that the supersymmetric may be bihamiltonian for particular values $k \mathrm{~s}$. Following Oevel and Popowicz [40] we assume $k=-1=k_{4}=-k_{3}=$ $-k_{2}=k_{1}$. Many of these $k_{i} \mathrm{~s}$ are chosen in such a way so that one can perform cancellation of certain terms.

Our goal is to construct the noncommutative analogue of the higher supersymmetric generalization of the super-KdV equation [36]. Thus, once again we use the modified definition of the Euler-Poincaré equation to obtain the dispersionless super-KdV equation.

Definition 3 The Euler-Poincare equation induced by the action of supersymmetric vector field $\operatorname{sect}\left(S^{1}\right)$ on its dual $\mathcal{F}_{-2}+\mathcal{F}_{-3 / 2}+\mathcal{F}_{-3 / 2}+\mathcal{F}_{-1}$ is defined as

$$
\begin{aligned}
& \left(u \xi^{-2}+\eta_{1} \xi^{-3 / 2}+\eta_{2} \xi^{-3 / 2}+w \xi^{-1}\right)_{t} \\
& \quad=a d_{\left(\frac{\delta H}{\delta u} \xi+\frac{\delta H}{\delta \eta_{1}} \xi^{1 / 2}+\frac{\delta H}{\delta \eta_{2}} \xi^{1 / 2}+\frac{\delta H}{\delta w}\right)}^{*}\left(u \xi^{-2}+\eta_{1} \xi^{-3 / 2}+\eta_{2} \xi^{-3 / 2}+w \xi^{-1}\right)
\end{aligned}
$$

3.1 Generalization of Souriau Cocycle to $N=2$ Superconformal Algebras

Let $\Phi_{i}$ and $\Psi_{i}$ be the odd parts of the super $\psi D\left(S^{1}\right)$. The nontrivial central extension of the Fermionic part of the super $\psi D\left(S^{1}\right)$ is:

$$
c_{\text {fermionic }}\left(\Phi_{i}, \Psi_{i}\right)=\int \operatorname{res}\left(\left[\ln \xi, \Phi_{i}(x)\right] \Psi_{i}\right) d x .
$$

Let us compute the pair of cocycles connected to the fermions.

$$
\begin{aligned}
c_{\text {fermionic }}\left(\phi_{i}(x) \xi^{1 / 2}, \psi_{i}(x) \xi^{1 / 2}\right) & =\int \operatorname{res}\left(\left[\ln \xi, \phi_{i}(x) \xi^{1 / 2}\right] \psi_{i} \xi^{1 / 2}\right) d x \\
& =\int \operatorname{res}\left(\left(\phi_{i}^{\prime} \xi^{-1 / 2}-\frac{1}{2} \phi_{i}^{\prime \prime} \xi^{-3 / 2}+\cdots\right) \psi_{i} \xi^{1 / 2}\right) \\
& =\int \operatorname{res}\left(\cdots-\frac{1}{2} \phi_{i}^{\prime \prime} \psi_{i} \xi^{-1}+\cdots\right)=\frac{1}{2} \int \phi_{i}^{\prime \prime} \psi_{i} d x .
\end{aligned}
$$

There exists another bosonic cocycle in $N=2$ superconformal algebra, given by

$$
\begin{aligned}
c_{\text {boson }}(v(x), w(x)) & =\int \operatorname{res}([\ln \xi, v(x)] \circ w(x)) \\
& =\int v^{\prime}(x) w(x) d x .
\end{aligned}
$$

Thus using the definition of logarithmic cocycle we derive the two cocycle of $N=2$ Neveu-Schwarz algebra. 
Proposition 2 The expression of $N=2$ superalgebra 2 cocycle in component form is given as

$$
\begin{aligned}
& \Omega\left(\left(f_{1} \frac{d}{d x}, \phi_{1}(d x)^{-1 / 2}, \phi_{2}(d x)^{-1 / 2}, f_{2}(x)\right),\left(g_{1} \frac{d}{d x}, \psi_{1}(d x)^{-1 / 2}, \psi_{2}(d x)^{-1 / 2} g_{2}(x)\right)\right) \\
& =\int_{S^{1}}\left(f_{1}^{\prime \prime} g_{1}^{\prime}+\phi_{1} \psi_{1}^{\prime \prime}+\phi_{2} \psi_{2}^{\prime \prime}-f_{2} g_{2}^{\prime}\right) d x .
\end{aligned}
$$

Thus $N=2$ Lie superalgebra can be extended by the two cycle $\Omega$. This allows us to compute the super-Hamiltonian operator for $N=2$ super $\mathrm{KdV}$ equation.

Proposition 3 The super-Hamiltonian operator $\mathcal{O}_{s k d v_{2}}$ corresponding to the action of the centrally extended $N=2$ Neveu-Schwarz algebra on its extended dual $\left(u(x) \xi^{-2}+\right.$ $\left.\eta_{i}(x) \xi^{-3 / 2}+w(x) \xi^{-1}, \mathbf{c}\right)$ yields

$$
\mathcal{O}_{s k d v_{2}}=\left(\begin{array}{cccc}
-\partial^{3}+2 u \partial+2 \partial u & \partial \eta_{1}+2 \eta_{1} \partial & \partial \eta_{2}+2 \eta_{2} \partial & 2 w \partial_{x} \\
2 \partial \eta_{1}+\eta_{1} \partial & \partial^{2}-u & \partial w+w \partial & -\eta_{2} \\
2 \partial \eta_{2}+\eta_{2} \partial & -w \partial-\partial w & \partial^{2}-u & \eta_{1} \\
\partial w & \eta_{2} & -\eta_{1} & \partial
\end{array}\right),
$$

where we have normalized the operator $\mathcal{O}$.

Thus we give alternative derivation of super-Hamiltonian operator, given by [40]. We consider the following Laberge-Mathieu [30] Hamiltonian function

$$
H=\frac{1}{2} \int\left(u^{2}-w w^{\prime}-\eta_{i} \eta_{i}^{\prime}+a u w^{2}-2 a w \eta_{1} \eta_{2}\right) d x
$$

to study flow on the orbits of $N=2$ superconformal algebra. Using bosonic superfield

$$
\Phi=\theta_{2} \theta_{1} u(x)+\theta_{1} \eta_{1}+\theta_{2} \eta_{2}+w(x)
$$

Hamiltonian $H$ can be rewritten as

$$
H=\frac{1}{2} \int d x d \theta_{1} d \theta_{2}\left(\Phi D_{1} D_{2} \Phi+\frac{a}{3} \Phi^{3}\right)
$$

Using the Hamiltonian operator $\mathcal{O}_{s k d v_{2}}$ and Hamiltonian $H$ we obtain $N=2$ super $\mathrm{KdV}$ equation, given by Labelle and Mathieu [see [30], (2.4), also citeop].

\section{Geodesic Flows on Group of Area Preserving Diffeomorphisms on Cyclider}

Groups of area-preserving diffeomorphisms and their Lie algebras play an important role in modern physics literature. It is known that in a suitable basis, the Lie algebra of the group $S D i f f(2)$ tends to that of $S U(N)$ as $N \rightarrow \infty$.

Using Adler's trace formula [1] we fix the ad-invariant quantity

$$
\operatorname{Tr} L=\int_{C} L d x d \xi
$$


Thus, we can define a weakly nondegenerate invariant inner product [3] on $\operatorname{sdiff}(2)$ by

$$
\langle L, M\rangle=\operatorname{Tr}(L M)=\int_{C} L M d x d \xi \quad L, M \in \operatorname{sdiff}(2) .
$$

The Lie-Poisson bracket on sdiff (2) is given by

$$
\{\{f, g\}\}(\alpha)=\left\langle\alpha,\left\{\frac{\delta f}{\delta \alpha}, \frac{\delta g}{\delta \alpha}\right\}\right\rangle,
$$

where $\frac{\delta f}{\delta \alpha}$ denotes the Frechét derivative. Here we have used the double curly bracket notations from Bloch et al. [3].

\subsection{Geodesic Flow with Respect to $H^{1}$-Norm}

We study geodesic flow on the area preserving diffeomorphism group with respect to $H^{1}$-Sobolev norm on the $\operatorname{siff}(\mathcal{A})$ algebra. It is defined by

$$
\langle L, M\rangle=\int_{C} L M d x d \xi+\int_{C} L^{\prime} M^{\prime} d x d \xi \quad L, M \in \operatorname{sdiff}(2),
$$

where $L^{\prime}$ denotes derivatives with respect to both $x$ and $\xi$. But since all $L$ and $M$ are polynomial $\xi$. Hence for all practical purposes it boils down to

$$
\langle L, M\rangle=\int_{C} L M d x d \xi+v \int_{C} L_{x} M_{x} d x d \xi \quad v \in \mathbb{R} .
$$

In other words / always means the derivative with respect to $x$.

Let us compute again the coadjoint action:

Lemma 2 The coadjoint action with respect to $H^{1}$ metric is given by

$$
\left.a d_{F}^{*}(G)\right|_{H^{1}}=\left(1-v \partial^{2}\right)^{-1}\left\{\left(F,\left(1-v \partial^{2}\right) G\right\} .\right.
$$

Proof We start from

$$
\begin{aligned}
\langle F,\{G, H\}\rangle_{H^{1}} & =\int_{C} F^{\prime}\{G, H\}^{\prime} d x d \xi+\int_{C} F\{G, H\} d x d \xi \\
& =\int_{C}\left\{F^{\prime}, G^{\prime}\right\} H d x d \xi+\int_{C}\left\{F^{\prime}, G\right\} H^{\prime} d x d \xi \\
& =\int_{C}\left\{F,\left(1-v \partial^{2}\right) G\right\} H d x d \xi .
\end{aligned}
$$

Let us compute now the L.H.S. of (22)

$$
\begin{aligned}
\text { L.H.S. } & =\int_{\mathcal{A}}\left(a d_{G}^{*} F\right) H d x d \xi+\int_{\mathcal{A}}\left(a d_{G}^{*} F\right)^{\prime} H^{\prime} d x d \xi \\
& =\int_{\mathcal{A}}\left[\left(1-v \partial^{2}\right) a d_{G}^{*} F\right] H d x d \xi .
\end{aligned}
$$

Thus by equating the R.H.S. and L.H.S. we obtain the above formula. 
Therefore, we conclude:

Proposition 4 The Euler-Poincaré equation with respect to right invariant $H^{1}$ metric on the dual space of sdiff (2)* yields

$$
\frac{\partial m}{\partial t}=-a d_{\frac{\delta H}{\delta u}}^{*} m,
$$

where $m=\left(1-\partial^{2}\right) u$ and $H$ is Hamiltonian.

Thus we justify the replacement of $u$ by $m=u-u_{x x}$ for the computation of peakon type equations, and it is not at all a computational trick.

\subsection{Computation of $N=2$ Supersymmetric Camassa-Holm Equation}

In this section we derive $N=2$ super Camassa-Holm equation by replacing all variables by their Helmholtz counterparts. In our previous section we justify the replacement for the computation of peakon type equations.

Lemma 3 The Hamiltonian operator $\mathcal{O}_{\text {super }}$ corresponding to the $H^{1}$-action of $f(x) \xi+$ $\phi_{i}(x) \xi^{-1 / 2}+g(x) \in \operatorname{Vect}\left(S^{1 \mid 2}\right)$ on the dual space of the Neveu-Schwarz algebra on $u(x) \xi^{-2}+\eta_{i}(x) \xi^{-3 / 2}+w(x) \xi^{-1}$ yields

$$
\mathcal{O}_{\text {super }}=\left(1-v \partial_{x}^{2}\right)^{-1}\left(\begin{array}{cccc}
2 m \partial+2 \partial m & \partial \beta_{1}+2 \beta_{1} \partial & \partial \beta_{2}+2 \beta_{2} \partial & 2 n \partial_{x} \\
2 \partial \beta_{1}+\beta_{1} \partial & -m & \partial n+n \partial & -\beta_{2} \\
2 \partial \beta_{2}+\beta_{2} \partial & -n \partial-\partial n & -m & \beta_{1} \\
\partial w & \beta_{2} & -\beta_{1} & 0
\end{array}\right),
$$

where $m=u-v u_{x x}, \beta_{i}=\eta_{i}-v \eta_{i x x}$ and $n=w-v w_{x x}$.

Proof This follows directly from our previous results

$$
\begin{aligned}
& \left.a d_{f(x) \xi+\phi_{i}(x) \xi^{1 / 2}+g(x)}^{*}\left(u(x) \xi^{-2}+\eta_{i}(x) \xi^{-3 / 2}+w(x) \xi^{-1}\right)\right|_{H^{1}} \\
& =\left(1-v \partial^{2}\right)^{-1}\left\{f(x) \xi+\phi_{i}(x) \xi^{1 / 2}+g(x),\left(1-v \partial^{2}\right)\left(u(x) \xi^{-2}\right.\right. \\
& \left.\left.\quad+\eta_{i}(x) \xi^{-3 / 2}+w(x) \xi^{-1}\right)\right\}
\end{aligned}
$$

and the computation of the left hand side $\{.,$.$\} is similar to previous section.$

Once we transfer the Helmholtz operator to left hand side we express EP equation in the following framework.

Definition 4 The Euler-Poincare equation induced by the action of $N=2$ supersymmetric $\operatorname{Vect}\left(S^{1}\right)$ on its dual $\mathcal{F}_{-2}+\mathcal{F}_{-3 / 2}+\mathcal{F}_{-3 / 2}+\mathcal{F}_{-1}$ with respect to $H^{1}$ norm is defined as

$$
\left(m \xi^{-2}+\beta_{i} \xi^{-3 / 2}+w \xi^{-1}\right)_{t}=a d_{\left(\frac{\delta H}{\delta u} \xi+\frac{\delta H}{\delta \eta_{i}} \xi^{1 / 2}+\frac{\delta H}{\delta g}\right)}^{*}\left(m \xi^{-2}+\beta_{i} \xi^{-3 / 2}+w(x) \xi^{-1}\right),
$$

where $m=u-v u_{x x}, \beta_{i}=\eta_{i}-v \eta_{i x x}$ and $n=w-v w_{x x}$ are the Helmholtz counter parts of $u, \eta_{i}$ and $w$ respectively. 
Proposition 5 The Euler-Poincaré flow with respect to $H^{1}$-metric on the dual space of $N=2$ Neveu-Schwarz algebra yields the $N=2$ super Camassa-Holm equation

$$
\begin{aligned}
m_{t}= & 4 m u_{x}+2 m_{x} u+4 a m w w_{x}+a m_{x} w^{2}-3 \eta_{i} \eta_{i}^{\prime \prime}+v \eta_{i}^{\prime} \eta_{i}^{\prime \prime \prime}+3 a\left(w \eta_{2} \beta_{1}+w \beta_{2} \eta_{1}\right)_{x} \\
& +2 a w\left(\beta_{1}^{\prime} \eta_{2}-\eta_{1} \beta_{2}^{\prime}\right)-2 n(x) w^{\prime \prime \prime}+2 a n(x)(u w)_{x}-2 a n(x)\left(\eta_{1} \eta_{2}\right)_{x} \\
\beta_{1 t}= & 3\left(\beta_{1} u+\frac{1}{2} a \beta_{1} w^{2}\right)_{x}-\beta_{1}^{\prime}\left(u+\frac{1}{2} a w^{2}\right)+m \eta_{1}^{\prime}+a m w \eta_{2}-2\left(n(x) \eta_{2}^{\prime}\right)_{x} \\
& +2\left(n w \eta_{1}\right)_{x}+n^{\prime}\left(\eta_{2}^{\prime}-a w \eta_{1}\right)+\beta_{2}\left(w^{\prime \prime}-a u w+a \eta_{1} \eta_{2}\right) \\
\beta_{2 t}= & 3\left(\beta_{2} u+\frac{1}{2} a \beta_{2} w^{2}\right)_{x}-\beta_{2}^{\prime}\left(u+\frac{1}{2} a w^{2}\right)+m \eta_{1}^{\prime}-a m w \eta_{1}+2\left(n(x) \eta_{1}^{\prime}\right)_{x} \\
& +2 a\left(n(x) w \eta_{2}\right)_{x}-n^{\prime}\left(\eta_{1}^{\prime}+a w \eta_{2}\right)+\beta_{1}\left(-w^{\prime \prime}+a u w-a \eta_{1} \eta_{2}\right) \\
n_{t}(x)= & \left(n u+\frac{1}{2} n w^{2}\right)_{x}-\beta_{2}\left(\eta_{1}^{\prime}+a w \eta_{2}\right)+\beta_{1}\left(\eta_{2}^{\prime}-a w \eta_{1}\right) .
\end{aligned}
$$

Proof We use the above definition for our proof.

Unfortunately there are not so much cancellation of terms due the existence Helmholtz functions are absence of cocycle terms. These two play very important role to write the $N=2$ super $\mathrm{KdV}$ equation in a compact form.

\section{$4.3 N=2$ Supersymmetric Camassa-Holm Equation in Covariant Form}

Clearly one can see that the $N=2$ is bit cumbersome in component form. One easily express this in superfield form.

We define the supercircle $S^{1 / 2}$ in terms of its superalgebra of functions denoted by $C_{\mathbb{C}}^{\infty}\left(S^{1 \mid 2}\right)$ consisting of elements of the form

$$
F(X)=f(x)+\theta_{1} \phi_{1}(x)+\theta_{2} \phi_{2}(x)+\theta_{1} \theta_{2} g(x),
$$

where $f, g$ and $\phi_{i}$ are smooth functions on $S^{1}$. Here $X$ stands for the triplet $\left(x, \theta_{1}, \theta_{2}\right)$, we assume $x$ is an arbitrary parameter on $S^{1}$ and $\theta_{1}$ and $\theta_{2}$ are formal Grassmann coordinates. These anticommuting variables satisfy

$$
\theta_{1} \theta_{2}=-\theta_{2} \theta_{1}, \quad \theta_{1}^{2}=\theta_{2}^{2}=0 .
$$

Identifying the element $\left(w, \eta_{2}, \eta_{1}, u\right) \in \operatorname{Vect}\left(S^{1 \mid 2}\right)^{*}$ with the odd (parity) dual super element

$$
U=w+\theta_{1} \eta_{2}+\theta_{2} \eta_{1}+\theta_{1} \theta_{2} u
$$

In the superfield form the (second) super Hamiltonian operator of $N=2$ super CamassaHolm equation can be rewritten as

$$
\mathcal{O}_{\text {super }}^{\prime}=\left(1-v \partial_{x}^{2}\right)^{-1}\left(2 \partial_{x} M+2 M \partial_{x}-\left(D_{i} M\right) D_{i}\right),
$$

where the superfield $M=U-v U_{x x}$. This allows us to express the $N=2$ supersymmetric Camassa-Holm equation in a more compact form. 
Proposition 6 The Euler-Poincaré flow on the dual of $N=2$ superconformal algebra with respect to $H^{1}$ norm yields the $N=2$ super Camassa-Holm equation

$$
M_{t}+2\left(M D_{1} D_{2} U\right)_{x}-\epsilon_{i j} D_{i} M D_{j} U_{x}+a\left(M U^{2}\right)_{x}+a M\left(U^{2}\right)_{x}-a D_{i} M D_{i} U=0 .
$$

Moreover this supersymmetric equation is equivalent to (26-29).

Proof We use the Hamiltonian equation

$$
U_{t}=\mathcal{O}_{\text {super }}^{\prime}\left(\frac{\delta H}{\delta U}\right)
$$

where $H=\frac{1}{2} \int d X\left(U D_{1} D_{2} U+\frac{a}{3} U^{3}\right)$. Thus we obtain

$$
M_{t}=\left(2 \partial_{x} M+2 M \partial_{x}-\left(D_{i} M\right) D_{i}\right)\left(D_{1} D_{2} U+\frac{a^{2}}{2} U^{2}\right)
$$

to obtain our result.

\section{Euler-Poincaré Flow and (Super) $b$-Field Equation}

In this section we derive of the Degasperis-Procesi equation and $b$-field equation. The DP equation is considered to be the second member of the one parameter $b$-field family of partial differential equations, which is given by

$$
m_{t}=3 m u_{x}+m_{x} u, \quad m=u-u_{x x} .
$$

At first our goal is to derive this equation from the action of $\operatorname{Vect}\left(S^{1}\right)$ on tensor densities. It is clear that

$$
\left\{f \xi, g \xi^{-(b-1)}\right\}=\left(f g^{\prime}-(b-1) f^{\prime} g\right) \xi^{(b-1)} .
$$

Thus we consider the deformation of the algebra of vector fields

$$
\begin{aligned}
{[v, w]_{b} } & :=\frac{b}{2}[v, w]-\frac{b-2}{2}(v w)_{x} \\
& =v w_{x}-(b-1) v_{x} w
\end{aligned}
$$

We note that the deformation is symmetric and a total divergence, reminiscent of the Dorfman bracket. This $b$-bracket allows interpretation as an action of $\operatorname{Vect}\left(S^{1}\right)$ on $\mathcal{F}_{(b-1)}\left(S^{1}\right)$. For $b=2$ this is merely the vector field action corresponding to the Lie bracket. The $b$-bracket is clearly not skewsymmetric. However, it has several interesting properties.

There exists a pairing [14]

$$
\mathcal{F}_{\lambda} \otimes \mathcal{F}_{1-\lambda} \rightarrow \mathbb{R}
$$

given by

$$
\left\langle a(x)(d x)^{\lambda}, b(x)(d x)^{1-\lambda}\right\rangle=\int a(x) b(x) d x .
$$

Therefore the above pairing allows us to identify the dual of $\mathcal{F}_{(b-1)}\left(S^{1}\right)$ with $\mathcal{F}_{-b}\left(S^{1}\right)$. 
Definition 5 The generalized EPDiff flow induced by the action $\operatorname{Vect}\left(S^{1}\right)$ on $\Psi D\left(S^{1}\right)$ space is defined as

$$
m_{t}=\left\{\frac{\delta H}{\delta u} \xi, m \xi^{-b}\right\},
$$

where $m=u-v u_{x x}$ is the standard Helmholtz operator acting on $u$.

It is clear that (37) is equivalent to Hamiltonian flow on the tensor densities $\mathcal{F}_{-b}$ of weight $b$ (or $b$-forms) given by

$$
u_{t}=\mathcal{O}_{b}^{H^{1}} \frac{\delta H}{\delta u}
$$

Theorem 3 Suppose we define Hamiltonian $H=\frac{1}{2} \int_{S^{1}} u^{2} d x$. The Euler-Poincaré equation for the right invariant $H^{1}$ metric on tensor densities $\mathcal{F}_{-b}$ (the dual space of b algebra) yields the $b$-field equation

$$
m_{t}=m_{x} u+b m u_{x}, \quad m=u-v u_{x x} .
$$

Proof The action of $\frac{\delta H}{\delta u}$ on $m \xi^{-b}$ is given by

$$
\begin{aligned}
& a d_{\frac{\delta H}{\delta u}(x) \xi} m(x) \xi^{-b} \\
& =-\left(\frac{\partial}{\partial \xi}\left(\frac{\delta H}{\delta u} \xi\right) \frac{\partial}{\partial x}\left(m \xi^{-b}\right)\right)-\frac{\partial}{\partial x}\left(\frac{\delta H}{\delta u} \xi\right) \frac{\partial}{\partial \xi}\left(m \xi^{-b}\right) \\
& =-\left(\frac{\delta H}{\delta u} m^{\prime}+b\left(\frac{\delta H}{\delta u}\right)^{\prime} m\right) \xi^{-b-1} .
\end{aligned}
$$

Substituting $\frac{\delta H}{\delta u}=u$ we obtain our result.

This equation was introduced in Degasperis, Holm and Hone [7, 8] based on Degasperis and Procesi [6] who singled out the cases $b=2$ Camassa-Holm equation and $b=3$ Degasperis-Procesi (DP) equation. Hamiltonian structure from the Euler-Poincare formalism is given in [19].

\subsection{Supersymmetric $b$-Field Equation}

Let us study the fermionic (i.e. $N=1$ supersymmetry) extension of the $b$-field equation. Consider the space of $-(2 b-1) / 2-$ tensor densities $\mathcal{F}_{-2 b-1 / 2}$. There exists a natural action of Lie superalgebra $\operatorname{Vect}\left(S^{1}\right) \oplus \mathcal{F}_{1 / 2}$ on $\mathcal{F}_{b} \oplus \mathcal{F}_{-2 b-1 / 2}$.

In our case, the super $b$ bracket is the deformation of the Neveu-Schwarz superconformal algebra, consisting of pairs $(u(x), \phi(x))$, where $u$ is a bosonic field and $\phi(x)$ is a fermionic field. The bracket is defined by

$$
[(u, \phi),(v, \xi)]_{b}=\left(u v_{x}-(b-1) u_{x} v, u \xi_{x}-\left(b-\frac{3}{2}\right) u_{x} \xi-(b-1) v \phi_{x}+\frac{1}{2} v_{x} \phi\right)
$$

Consideration of the supersymmetrisation of this algebra then opens the door to the construction of supersymmetric extensions of the $b$-field equations. 
Remark A vector field $X_{f}$ on $S^{1 \mid 1}$, for any $f \in C_{\mathbb{C}}^{\infty}\left(S^{1 \mid 1}\right)$, is said to be contact if it preserves the contact distributions. The contact bracket is defined by $\left[X_{f}, X_{g}\right]=X_{\{f, g\}}$, where the space $C_{\mathbb{C}}^{\infty}\left(S^{1 \mid 1}\right)$ is thus equipped with a Lie superalgebra structure given by

$$
\{f, g\}=f g^{\prime}-f^{\prime} g+(-1)^{p(f) p(g)+1} \frac{1}{2} D(f) D(g),
$$

where $D=\frac{\partial}{\partial \theta}+\theta \frac{\partial}{\partial x}$. The super $b$ bracket that we are considering belongs to a class of generalized contact bracket [13] which extends to densities of arbitrary weight:

$$
\{., .\}: \mathcal{F}_{\lambda}\left(S^{1 \mid 1}\right) \otimes \mathcal{F}_{\mu}\left(S^{1 \mid 1}\right) \rightarrow \mathcal{F}_{\lambda+\mu+1}\left(S^{1 \mid 1}\right),
$$

explicitly given by

$$
\{f, g\}=\lambda f g^{\prime}-\mu f^{\prime} g+(-1)^{p(f) p(g)+1} \frac{1}{2} D(f) D(g) .
$$

Lemma 4 The Hamiltonian operator $\mathcal{O}_{b}$ corresponding to the action of the Neveu-Schwarz algebra on $m(x) \xi^{-b}+\beta(x) \xi^{-2 b-1 / 2} \in \Psi D\left(S^{1}\right)$ yields

$$
\mathcal{O}=-\left(\begin{array}{c|c}
\partial m+(b-1) m \partial & \frac{1}{2} \partial \beta+(b-1) \beta \partial \\
\hline \partial \beta+\frac{2 b-3}{2} \beta \partial & \frac{1}{2} m
\end{array}\right)
$$

Proof It follows straight away from

$$
\begin{aligned}
& a d_{f(x) \xi+\phi(x) \xi^{1 / 2}}^{*}\left(m(x) \xi^{-b}+\beta(x) \xi^{-2 b-1 / 2}\right) \\
& =\left\{f(x) \xi, m(x) \xi^{-b}+\beta(x) \xi^{-2 b-1 / 2}\right\}+\left\{\phi(x) \xi^{1 / 2}, \beta(x) \xi^{-2 b-1 / 2}\right\} \\
& \quad-\frac{1}{2} \phi(x) m(x) \xi^{-3 / 2},
\end{aligned}
$$

where the last expression follows from the definition of zeroth operator given by Grozman and we have chosen $k=-\frac{1}{2}$.

Proposition 7 Let $\frac{\delta H}{\delta u}=2 u$ and $\frac{\delta H}{\delta \eta}=8 \eta_{x}$. The Euler-Poincaré flow on $\mathcal{F}_{-b}+\mathcal{F}_{-2 b-1 / 2}$, yields the supersymmetric $b$-field equation

$$
\begin{aligned}
m_{t} & =2 m_{x} u+2 b m u_{x}+4(2 b-1) \beta \eta_{x x}+4 \beta_{x} \eta_{x} \\
\beta_{t} & =2 \beta_{x} u+4 m \eta_{x}+(2 b-1) \beta u_{x} .
\end{aligned}
$$

Proof It is clear that $\left(\frac{\delta H}{\delta u}, \frac{\delta H}{\delta \eta}\right) \in \operatorname{Vect}\left(S^{1 \mid 1}\right)$. Thus computing the (coadjoint) action $a d_{\frac{\delta H}{\delta u}(x) \xi+\frac{\delta H}{\delta \eta} \xi^{1 / 2}}^{*}\left(m(x) \xi^{-b}+\beta(x) \xi^{-2 b-1 / 2}\right)$ for $\frac{\delta H}{\delta u}=2 u$ and $\frac{\delta H}{\delta \eta}=8 \eta_{x}$ we obtain our desired result.

\section{Noncommutative Analogue of the Kuper Camassa-Holm and Supersymmetric $b$-Field Equations}

Finally in this section we propose another construction of noncommutative (or Moyal deformed) integrable systems. In particular, we demonstrate the derivation of noncommutative 
super peakon type systems. The main idea of this construction is to replace Poisson action by Moyal action of vector fields $\operatorname{Vect}\left(S^{1}\right)\left(\operatorname{or} \operatorname{Vect}\left(S^{1 \mid 1}\right)\right.$ on the space of pseudodifferential symbols.

\subsection{Construction of Noncommutative Bosonic Systems}

All the equations described in this article can be $\star$ deformed using following rules:

(A) All Poisson brackets should be replaced by Moyal brackets [38] defined by:

$$
\{F, G\}_{\text {Moyal }}:=\frac{F \star G-G \star F}{\kappa} .
$$

(B) The derivatives act on the $\star$-deformed space in a usual way.

We will study two types of systems here-one is purely bosonic ( or ordinary) system and other one is $N=1$ supersymmetric system.

The bosonic part can be quantized by using the Moyal product

$$
f \star_{M} g=f \exp \left[\frac{i \hbar}{2}\left(\overleftarrow{\partial_{q}} \overrightarrow{\partial_{p}}-\overleftarrow{\partial_{p}} \overrightarrow{\partial_{q}}\right)\right] g
$$

The Moyal star product replaces the ordinary product between functions on the phase space.

Definition-Proposition 1 Let $d H$ be the gradient of the Hamiltonian. In the noncommutative case Vect $\left(S^{1}\right)$ acts on the $\star$-deformed dual embedded in $\Psi D\left(S^{1}\right)$ is given by

$$
\begin{aligned}
u_{t} & =\left\{d H(x) \xi, u(x) \xi^{-2}\right\}_{\text {Moyal }}+\frac{1}{6}(d H)^{\prime \prime \prime} \xi^{-2} \\
& =\left(d H \star u^{\prime}+2 d H^{\prime} \star u+\frac{1}{6} d H^{\prime \prime \prime}\right) \xi^{-2}
\end{aligned}
$$

Corollary 1 Suppose $H=\frac{1}{2} \int_{S^{1}} u^{2} d x$. The Moyal deformed Euler-Poincaré flow on $\mathcal{F}_{-2}$ yields the noncommutative $K d V(n c K d V)$ equation

$$
u_{t}=u \star u_{x}+2 u_{x} \star u+u_{x x x} .
$$

Proposition 8 Let $d H$ be the gradient of the Hamiltonian with respect to Helmholtz function $m$. Let $\operatorname{Vect}\left(S^{1}\right)$ acts on the $\star$-deformed space of tensor densities $\mathcal{F}_{-b}$ embedded in $\Psi D\left(S^{1}\right)$. The noncommutative analogue of the EPDiff flow is given by

$$
\begin{aligned}
m_{t} & =\left\{d H(x) \xi, m(x) \xi^{-b}\right\}_{\text {Moyal }} \\
& =d H \star m^{\prime}+b d H^{\prime} \star m .
\end{aligned}
$$

Suppose $H=\int_{S^{1}}$ mudx, we obtain the noncommutative b-field equation

$$
m_{t}+u \star m_{x}+b u_{x} \star m=0
$$

Proof Straightforward. 


\subsection{Construction of Noncommutative $N=1$ Super $b$-Field Systems}

Noncommutativity in superspace naturally arise in string theory in several contexts. Imposing the worldsheet supersymmetry to the noncommutativity relation of the spacetime creates $\star$ products between the boson-boson, boson-fermion and fermion-fermion fields. It is known that the $\star$ product of two superfields is a superfield.

In this study the Moyal deformed super $b$-field equation using results of noncommutative superspaces $[12,24]$.

Here we invoke a generalization of Moyal-Weyl deformation to functions on super-phase space. This algebraic structure corresponds to the quantization of systems with both, bosonic and fermionic degrees of freedom. The fermionic variables involves a $\star$-product that is given by

$$
\phi \star_{C} \psi=\phi \exp \left[\frac{i \hbar}{2}\left(\overleftarrow{\partial_{\theta_{i}}} \overrightarrow{\partial_{\theta_{i}}}\right)\right] \psi
$$

This star product is called the Clifford star product because it leads to a cliffordization of the Grassmann algebra of the odd coordinates $\theta_{i}$. Moreover, the star anticommutator is given by

$$
\left\{\theta_{i}, \theta_{j}\right\}_{C}=\theta_{i} \star_{C} \theta_{j}+\theta_{j} \star_{C} \theta_{i}=\hbar \delta_{i j} .
$$

Proposition 9 The Euler-Poincaré flow with respect to $H^{1}$-metric on the Moyal deformed dual space of Neveu-Schwarz algebra yields the noncommutative Kupershmidt-CamassaHolm equation

$$
\begin{array}{r}
m_{t}+4 u_{x} \star m+2 u \star m_{x}+4 \eta_{x} \star \beta_{x}+12 \eta_{x x} \star \beta=0 \\
\beta_{t}+2 u \star \beta_{x}+3 u_{x} \star \beta+4 \eta_{x} \star m=0
\end{array}
$$

for (super) Hamiltonian $\frac{\delta H}{\delta u}=2 u$ and $\frac{\delta H}{\delta \eta}=8 \eta_{x}$.

Proof The Euler-Poincare equation induced by the action of Moyal deformed supersymmetric $\operatorname{Vect}\left(S^{1}\right)$ on its dual $\mathcal{F}_{-2}+\mathcal{F}_{-3 / 2}$ is given by

$$
\begin{aligned}
\left(m \xi^{-2}+\tilde{\eta} \xi^{-3 / 2}\right)_{t} & \\
= & \left\{\frac{\delta H}{\delta u} \xi, u(x) \xi^{-2}+\eta \xi^{-3 / 2}\right\}_{\text {Moyal }}+\left\{\frac{\delta H}{\delta \eta} \xi^{1 / 2}, \eta \xi^{-3 / 2}\right\}_{\text {Moyal }} \\
& +\frac{1}{2} \frac{\delta H}{\delta \eta} m(x) \star \xi^{-3 / 2}
\end{aligned}
$$

Therefore, using Hamiltonian $\frac{\delta H}{\delta u}=2 u$ and $\frac{\delta H}{\delta \eta}=8 \eta_{x}$ we obtain our desired result.

Similarly one can compute the noncommutative version of supersymmetric $b$-field equation.

Proposition 10 The Euler-Poincaré flow with respect to $H^{1}$-metric on $\star$-deformed $\mathcal{F}_{-b}+$ $\mathcal{F}_{-\frac{2 b-1}{2}}$ yields the Moyal deformed supersymmetric $b$-field equation

$$
\begin{array}{r}
m_{t}+2 b u_{x} \star m+2 u \star m_{x}+4 \eta_{x} \star \beta_{x}+4(2 b-1) \eta_{x x} \star \beta=0 \\
\beta_{t}+2 u \star \beta_{x}+(2 b-1) u_{x} \star \beta++4 \eta_{x} \star m=0
\end{array}
$$


Obviously, the best way to consider such deformations through the introduction of the (super) Poisson bracket between two superfields and consider the Moyal-Weyl star product of superfields.

\section{Outlook}

In the present paper, we have constructed various noncommutative integrable and superintegrable systems in $(1+1)$ through embedding of vector fields and its dual on the space of pseudodifferential symbols on $S^{1}$. In this process we have tacitly moved the coadjoint action to Poisson action. We have used Grozman's method coupled with the anticommutativity properties of fermions to compute these actions. Then replacing the Poisson action by Moyal action we have constructed the noncommutative or Moyal deformed integrable systems. We claim that this method is much more elegant and geometrical than previously known methods. It would be nice to formulate the solutions of these equations-hope one should be able implement the methods developed by Takasaki [46] to study the geometry of the corresponding Riemann-Hilbert problem in some Moyal algebra valued loop group.

Acknowledgements This paper is the vastly modified version of paper presented in Noncommutative Geometry and Physics workshop at Newton Institute, September 3-8, 2006. Hence author would like to thank the organizers of this workshop. He is extremely grateful to Valentin Ovsienko and Chandrasekhar Devchand for numerous explanations and correspondences. He is also particularly grateful to Jürgen Jost, Giovanni Landi, George Wilson and Shahn Majid for their interest. He is particularly grateful to Peter Gilkey for valuable suggestions. Finally author expresses grateful thanks to MPI-MIS for gracious hospitality.

\section{References}

1. Adler, M.: On a trace functional for formal pseudo-differential operators and the symplectic structure of the Korteweg de Vries type equations. Invent. Math. 50, 219-248 (1979)

2. Aschieri, P., Blohmann, C., Meyer, F., Schupp, P., Wess, J.: A gravity theory on noncommutative spaces. Class. Quantum Gravity 22(17), 3511-3532 (2005)

3. Bloch, A., Flaschka, H., Ratiu, T.: The Toda PDE and the geometry of the diffeomorphism group of the annulus. In: Mechanics Day, Waterloo, ON, 1992. Fields Inst. Commun., vol. 7, pp. 57-92. Am. Math. Soc., Providence (1996)

4. Camassa, R., Holm, D.D.: A completely integrable dispersive shallow water equation with peaked solutions. Phys. Rev. Lett. 71, 1661-1664 (1993)

5. Connes, A.: Noncommutative Geometry. Academic Press, San Diego (1994)

6. Degasperis, A., Procesi, M.: Asymptotic integrability. In: Symmetry and Perturbation Theory (Rome 1998), pp. 23-37. World Scientific, River Edge (1999)

7. Degasperis, A., Holm, D.D., Hone, A.N.W.: A new integrable equation with peakon solutions. In: NEEDS 2001 Proceedings. Theoret. Math. Phys., vol. 133, pp. 170-183 (2002)

8. Degasperis, A., Holm, D.D., Hone, A.N.W.: Integrable and non-integrable equations with peakons. In: Nonlinear Physics: Theory and Experiment, II, Gallipoli, 2002, pp. 37-43. World Scientific, River Edge (2003)

9. Devchand, C., Schiff, J.: The supersymmetric Camassa-Holm equation and geodesic flow on the superconformal group. J. Math. Phys. 42(1), 260-273 (2001)

10. Dimakis, A., Müller-Hoissen, F.: Moyal deformation, Seiberg-Witten maps, and integrable models. Lett. Math. Phys. 54(2), 123-135 (2000)

11. Faddeev, L.D., Takhtajan, L.A.: Hamiltonian Methods in the Theory of Solitons. Addison-Wesley, Reading (1987)

12. Ferrara, S., Lledó, M.: Some aspects of deformations of supersymmetric field theories. J. High Energy Phys. 5, Paper 8, 22 pages (2000)

13. Gargoubi, H., Ovsienko, V.: Supertransvectants and symplectic geometry. arXiv:0705.1411v1 (2007)

14. Gargoubi, H., Mathonet, P., Ovsienko, V.: Symmetries of modules of differential operators. J. Nonlinear Math. Phys. 12(3), 348-380 (2005) 
15. Grozman, P.: Invariant bilinear differential operators. Preprint ESI 1114 (2001). Available via http://www.esi.ac.at

16. Guha, P.: Moyal deformation of KdV and Virasoro action. J. Phys. Soc. Jpn. 73-10, 2662-2666 (2004)

17. Guha, P.: $\operatorname{Vect}\left(S^{1}\right)$ action on pseudodifferential symbols on $S^{1}$ and (noncommutative) hydrodynamic type systems. J. Nonlinear Math. Phys. 13(4), 549-565 (2006)

18. Guha, P.: Noncommutative integrable systems and diffeomorphism on quantum spaces. Class. Quantum Gravity 24, 497-506 (2007)

19. Guha, P.: Euler-Poincaré formalism of (two component) Degasperis-Procesi and Holm-Staley systems. J. Nonlinear Math. Phys. 14(3), 390-421 (2007)

20. Guha, P.: Supersymmetric Kuper Camassa-Holm equation and geodesic flow: a novel approach. Int. J. Geom. Methods Mod. Phys. 5(1), 1-16 (2008)

21. Hamanaka, M.: Commuting flows and conservation laws for noncommutative Lax hierarchies. hep-th/0311206 (2003)

22. Hamanaka, M., Toda, K.: Towards noncommutative integrable systems. Phys. Lett. A 316(1-2), 77-83 (2003). hep-th/0211148

23. Harvey, J.A.: Komaba lectures on noncommutative solitons and D-branes. hep-th/0102076 (2001)

24. Henselder, P., Hirshfeld, A.C., Spernat, T.: Star products and quantum groups in quantum mechanics and field theory. Ann. Phys. 308(1), 311-328 (2003)

25. Holm, D.D., Marsden, J.E., Ratiu, T.S.: The Euler-Poincaré equations and semidirect products with applications to continuum theories. Adv. Math. 137(1), 1-81 (1998)

26. Khesin, B., Zakharevich, I.: Poisson-Lie group of pseudodifferential symbols and fractional KP-KdV hierarchies. C. R. Acad. Sci. Paris Sér. I Math. 316(6), 621-626 (1993)

27. Kirillov, A.: The orbit method, I and II: Infinite-dimensional Lie groups and Lie algebras. Contemp. Math. 145, 33-63 (1993)

28. Kravchenko, O.S., Khesin, B.A.: A central extension of the algebra of pseudodifferential symbols. Funct. Anal. Appl. 25(2), 152-154 (1991)

29. Kupershmidt, B.: Quantizations and integrable systems. Lett. Math. Phys. 20(1), 19-31 (1990)

30. Labelle, P., Mathieu, P.: A new $N=2$ supersymmetric Korteweg-de Vries equation. J. Math. Phys. 32(4), 923-927 (1991)

31. Lechtenfeld, O.: Noncommutative solitons. hep-th/0600341 (2006)

32. Lechtenfeld, O.: Supersymmetric noncommutative solitons. hep-th/070735221 (2007)

33. Legaré, M.: Noncommutative generalized NS and super matrix KdV systems from a noncommutative version of (anti-) selfdual Yang-Mills equations. hep-th/0012077 (2000)

34. Leites, D., Kochetkov, Yu., Weintrob, A.: New invariant differential operators on supermanifolds and pseudo-(co)homology. In: General Topology and Applications, Staten Island, NY, 1989. Lecture Notes in Pure and Appl. Math., vol. 134, pp. 217-238. Dekker, New York (1991)

35. Majid, S.: A Quantum Groups Primer. London Mathematical Society Lecture Note Series, vol. 292. Cambridge University Press, Cambridge (2002)

36. Manin, Yu.I., Radul, A.O.: A supersymmetric extension of the Kadomtsev-Petviashvili hierarchy. Commun. Math. Phys. 98, 65-77 (1988)

37. Misiolek, G.: A shallow water equation as a geodesic flow on the Bott-Virasoro group. J. Geom. Phys. 24, 203-208 (1998)

38. Moyal, J.E.: Quantum mechanics as a statistical theory. Proc. Camb. Philos. Soc. 45, 99-124 (1949)

39. Nekrasov, N., Schwarz, A.: Instantons on noncommutative $\mathbf{R}^{4}$, and $(2,0)$ superconformal sixdimensional theory. Commun. Math. Phys. 198(3), 689-703 (1998)

40. Oevel, W., Popowicz, Z.: The bi-Hamiltonian structure of fully supersymmetric Korteweg-de Vries systems. Commun. Math. Phys. 139(3), 441-460 (1991)

41. Ovsienko, V., Khesin, B.: KdV super equation as an Euler equation. Funct. Anal. Appl. 21, 329-331 (1987)

42. Ovsienko, V., Roger, C.: Deforming the Lie algebra of vector fields on $S^{1}$ inside the Poisson algebra on $\dot{T}^{*} S^{1}$. Commun. Math. Phys. 198(1), 97-110 (1998)

43. Ovsienko, V., Roger, C.: Deforming the Lie algebra of vector fields on $S^{1}$ inside the Lie algebra of pseudodifferential symbols on $S^{1}$. In: Differential Topology, Infinite-Dimensional Lie Algebras, and Applications. Amer. Math. Soc. Transl. Ser. 2, vol. 194, pp. 211-226. Am. Math. Soc., Providence (1999)

44. Seiberg, N., Witten, E.: String theory and noncommutative geometry. J. High Energy Phys. 9, Paper 32, 93 pages (1999). (Electronic)

45. Szabo, R.J.: D-branes in noncommutative field theory. hep-th/0512054 (2005)

46. Takasaki, K.: Dressing operator approach to Moyal algebraic deformation of selfdual gravity. J. Geom. Phys. 14, 111-120 (1994)

47. Varadarajan, V.S.: Supersymmetry for Mathematicians: An Introduction. Courant Lecture Notes in Mathematics, vol. 11. New York University, Courant Institute of Mathematical Sciences/Am. Math. Soc., New York/Providence (2004) 